\title{
Des femmes dans leurs rôles : pour une relecture des guerres civiles à Rome (Ier siècle av. J.-C.)
}

Jean-Marie PAILLER

\section{(2) OpenEdition}

1 Journals

\section{Édition électronique}

URL : https://journals.openedition.org/clio/408

DOI : $10.4000 /$ clio.408

ISSN : 1777-5299

Éditeur

Belin

Édition imprimée

Date de publication : 1 avril 1997

ISBN : 2-85816-323-5

ISSN : $1252-7017$

\section{Référence électronique}

Jean-Marie PAILLER, « Des femmes dans leurs rôles : pour une relecture des guerres civiles à Rome (ler siècle av. J.-C.) », Clio. Histoire, femmes et sociétés [En ligne], 5 | 1997, mis en ligne le 01 janvier 2005, consulté le 22 avril 2022. URL : http://journals.openedition.org/clio/408; DOI : https://doi.org/ 10.4000/clio.408

Ce document a été généré automatiquement le 22 avril 2022.

Tous droits réservés 


\title{
Des femmes dans leurs rôles : pour une relecture des guerres civiles à Rome (Ier siècle av. J.-C.)
}

\author{
Jean-Marie PAILLER
}

1 «[Catilina] rallia à sa cause nombre d'hommes de toute espèce, et même quelques femmes qui avaient d'abord demandé à la galanterie les moyens de subvenir à leur prodigalité, puis, quand l'âge eut réduit leurs gains mais non leurs besoins, avaient contracté d'énormes dettes. Par elles Catilina croyait pouvoir soulever les esclaves urbains, incendier la ville, s'adjoindre leurs maris ou les assassiner.

2 De leur nombre était Sempronia, qui avait déjà commis plus d'un méfait d'une audace toute virile. Par sa naissance et sa beauté, par son mari, par ses enfants, cette femme n'avait eu qu'à se louer de la fortune; instruite également aux lettres grecques et latines, elle jouait de la cithare et dansait avec plus d'art qu'il ne convient à une honnête femme, sans compter bien d'autres talents, auxiliaires de la débauche (...) Brûlante de sensualité, il lui arrivait plus souvent de solliciter les hommes que d'en être sollicitée. Bien des fois auparavant elle avait trahi sa parole, nié solennellement une dette, trempé dans des meurtres; la débauche et la misère avaient précipité sa chute. Pourtant elle avait un esprit assez distingué : elle savait tourner les vers, trouver le mot pour rire, tenir un langage tantôt modeste, tantôt tendre ou provocant ; bref il y avait en elle autant de finesse que de charme... »

3 Sur la perception du rôle ${ }^{1}$ des femmes dans le climat et les intrigues des guerres civiles de la fin de la République romaine, peu d'œuvres anciennes nous en disent autant que la plume informée, acérée mais finalement nuancée et distanciée de Salluste (Conjuration de Catilina, XXIV, 3 - XXV, 5). Le portrait de Sempronia n'y apparaît pas seulement comme celui d'une figure individuelle, mais d'une catégorie, mieux, d'un type : le type des femmes libres, riches et dévoyées dont le chef de la conspiration, en 63 av. J.-C., compte se servir pour mener à bien certains de ses projets. «Seconds couteaux » ("même quelques femmes... ») et simples instruments (per eas, "par elles ») de la guerre civile en gestation, ces femmes ne s'en comportent pas moins en acteurs et 
- c'est cela que Salluste met en valeur, et qui doit scandaliser - en acteurs autonomes. De haute naissance, aisées (même si elles sont couvertes de dettes, donc en parfaite harmonie de situation et de motivation avec Catilina et ses comparses), instruites et " hellénisées », elles poursuivent des objectifs personnels, savent manipuler époux et amants, peuvent entraîner, dans l'immense Rome de la République agonisante, les domesticités serviles qu'elles gouvernent de fait, et n'hésitent devant rien, pas plus le meurtre que le faux serment. Bref, elles font preuve d'une « audace toute virile » (uirilis audacia) : comble de l'abomination et symbole de ce renversement de toutes les valeurs qui fait, aux yeux des témoins de l'époque, le fond même des guerres civiles ${ }^{2}$. Et pourtant, Salluste, qui les a côtoyées, reconnaît in fine "autant de finesse que de charme » (multae facetiae multusque lepos) à l'une au moins de ces femmes d'influence.

4 Comme tout portrait de femme, celui-ci ne prend sa valeur que rapporté au tableau plus général de ce sexe que l'historien latin met en scène. Une image très diversifiée se dessine de la sorte au long des chapitres XI à XV du Catilina. C'est d'abord cette remarque sur le goût effréné de l'argent: "comme s'il était imprégné de poisons maléfiques, ce vice effémine les âmes et les corps les plus virils» (corpus animumque uirilem effeminat, $\mathrm{XI}, 3)$. Ce vice est inverse et symétrique de celui qui a atteint Sempronia et ses pareilles, la "virilisation » (uirilis audaciae, XXV, 1). L'inversion, au sens le plus fort, des rôles sexuels est une caractéristique des situations de guerre civile ou de troubles internes, telles que nous les présente la tradition romaine. Produite, selon Salluste, par les conquêtes orientales de Sylla, au début du siècle, cette perversion généralise ses effets et gangrène la société romaine dans son ensemble : "les hommes se prostituaient comme des femmes [en fait ${ }^{3}$, l'alliance de mots et le choix du verbe en latin sont plus expressifs : uiri muliebria pati], les femmes offraient leur pudeur à tout venant » (XIII, 3). Pour l'historien latin, comme pour ses contemporains, ce climat imprègne l'ensemble de la période qui conduit des guerres civiles entre partisans de Marius et soutiens de Sylla, dans les années 80 av. J.-C., à celles qui opposent successivement, de 51 à 46, les pompéiens aux césariens, puis les "républicains " assassins de César (44) à ses héritiers Antoine et Octave, enfin ces derniers entre eux jusqu'à la bataille d'Actium (31) et jusqu'à ce jour de 27 av. J.-C. où Octave prend le nom d'Auguste et instaure de facto une monarchie.

Bien entendu, il est encore à Rome, même dans ce contexte, des femmes dignes de leur rang, et de la tradition. Celles-là, à en croire Salluste, ne sont pas coupables, mais victimes. Témoin, en premier lieu, les traces d'affolement collectif devant ce qui s'annonce en 63 comme une nouvelle guerre interne à la république, un affolement décrit dans des termes qui rappellent la panique féminine aux heures les plus sombres de l'invasion carthaginoise en Italie, un siècle et demi auparavant: «Les femmes, envahies par la peur de la guerre dont la puissance de la république les avait déshabituées, se frappaient la poitrine, tendaient vers le ciel des mains suppliantes, se lamentaient sur leurs petits enfants, multipliaient les questions, s'épouvantaient de tout, oubliant orgueil et plaisirs désespéraient d'elles-mêmes et de la patrie » (Catilina, XXXI, 3). Une autre victime emblématique, quoiqu'ap-paremment consentante, est la Vestale dévoyée par Catilina lui-même: l'accusation d'«amours criminelles", conduites contra ius fasque, " contrairement aux lois humaines et divines » $(\mathrm{XV}, 1)$, se laisse comparer à celle portée contre un autre grand adversaire de Cicéron, Clodius ${ }^{4}$. Elle prend toute sa gravité si l'on songe à ce que représentaient les prêtresses de Vesta, et à ce que signifiait pour elles-mêmes et pour Rome leur « inceste ». Dira-t-on que la rumeur sur cette liaison sacrilège remonte à la première jeunesse du conspirateur, et 
ne s'inscrit donc pas dans le contexte propre de la crise ? Ce serait oublier que la jeune fille, Fabia, était la demi-sœur de Térentia, femme de Cicéron. Quelle qu'ait pu être la réalité exacte des faits, le souvenir attesté par Salluste ne s'en était sûrement pas perdu dans l'esprit du consul sauveur de Rome en 63...

6 L'historien fait encore leur place à deux personnages féminins moins typés, donc moins soupçonnables d'avoir été travestis, sinon inventés, aux dépens de la vérité historique : Orestilla et Fulvia. Immédiatement après la Vestale, il évoque la seconde femme dans la vie de Catilina - ou la troisième, si l'on tient compte d'un premier mariage -, celle qui est au moment des événements son épouse. Cette Aurelia Orestilla, plutôt que criminelle, apparaît occasion de crime. On n'avait certes « rien trouvé d'autre à louer en elle que sa beauté » $(\mathrm{XV}, 2)$, mais c'est de lui-même, certes pour lui complaire mais non à sa demande, que Catilina aurait fait assassiner son propre fils, né d'un premier lit. Salluste fait de ce geste le point de départ du déchaînement de 63: «c'est même ce meurtre qui, selon moi, fut pour lui la principale raison de hâter son forfait » $(\mathrm{XV}, 3)$. Un peu plus loin, nous apprenons par Catilina en personne qu'Orestilla est très riche et dévouée, puisqu'elle aurait pu, avec sa fille, payer les dettes du clan entier (XXXV, 3). Mais le personnage, aux antipodes d'une Sempronia, demeure fondamentale-ment passif : son époux ne la mentionne en outre que pour la confier, l'heure des hostilités étant venue, au sénateur et ancien consul Q. Lutatius Catulus (XXXV, 6).

7 Fulvia, quant à elle, est à la fois active et «agie». Catilinienne repentie ou, comme on voudra, traîtresse à la cause, c'est elle qui dévoile régulièrement les plans des conjurés (XXIII, 3-4, XXVI, 2, XXVIII, 2). Deux choses frappent en elle : des mobiles strictement privés et très "féminins" (amour ou amour-propre bafoué, bavardage), un rôle d'intermédiaire fidèle entre son amant Curius et Cicéron (per Fulviam, XXVI, 3 et XXVIII, 2). Femme de noble origine (muliere nobili, XXIII, 3), elle entretient de longue date une liaison avec un aristocrate dépravé et déclassé, rayé de l' »album » sénatorial par les censeurs. Ce Curius, voulant l'impressionner, se vante de faire partie du complot. Elle en répand le bruit, et... « ce fut la cause principale du mouvement d'opinion qui poussa Cicéron au consulat» (XXIII, 5). En somme, comme pour le déclenchement des hostilités par Catilina, il faut " chercher la femme », mais dans les deux cas elle n'est qu'une cause involontaire.

8 Cette brève relecture du récit de Salluste nous a livré des portraits qui sont par trop à l'image de rôles pour que nous ayons la naïveté de les croire entièrement fidèles à la « réalité » historique. Comme il arrive à l'historien d'aujourd'hui lorsque manquent à l'appel les recoupements avec d'autres sources, notamment autres que littéraires, nous sommes contraints de prendre d'un bloc le document, où l'« information brute » est traversée de part en part par le travail de l'interprétation. N'allons pourtant pas nous plaindre de ne pouvoir trier le bon grain réaliste de l'ivraie, ou de l'ivresse, herméneutique. Des œuvres comme le Catilina, confronté en particulier avec ce témoignage encore plus marqué de « subjectivité » combattante que sont les Catilinaires de Cicéron, nous éclairent à la fois sur l'activité de personnages incontestablement réels et sur ce qu'en ont fait les auteurs, témoins diversement engagés. Ainsi se dégagent les traits d'un « imaginaire féminin » des guerres civiles.

Les Romains, comme l'a répété Georges Dumézil, "pensent nationalement et historiquement »- nous ajouterons : exemplairement, en termes de modèles. Rien ou presque de ce que disent de leur temps les écrivains de la fin de la République ne peut être saisi indépendamment de l'arrière-plan fourni par la tradition. C'est en fonction de 
celle-ci et par rapport à elle que personnages et épisodes de l'époque récente ou contemporaine prennent relief et sens. Il n'est donc pas inutile, afin de mieux saisir ce qui informe et nourrit historiquement l'imaginaire qui nous intéresse, de revenir tour à tour sur quatre moments de l'évolution romaine bien antérieurs à la crise finale de la République, mais constitutifs de la mémoire collective - quatre moments où la situation de Rome a pu s'apparenter à celle d'une guerre civile, et qui sont marqués par l'intervention de femmes. Seront successivement envisagés deux épisodes légendaires, puis deux autres qu'on peut qualifier d'historiques au sens, complexe et nuancé, que nous avons vu plus haut: les Sabines aux origines de l'unité civique (fondation de Rome, 754 av. J.-C.) ; la vertu de Lucrèce au point de départ de la «révolution républicaine » (509); Claudia Quinta, ou la concorde rétablie par la dissipation du soupçon (204); un contre-exemple, enfin, en 186: les bacchantes, menace de dissolution du système civico-familial.

10 1) Les Sabines enlevées par les compagnons de Romulus ont eu pour double destin de provoquer, bien involontairement, la guerre entre Sabins et Latins, puis d'y mettre un terme par leur intervention délibérée : «c'est alors que les Sabines, dont le rapt avait amené la guerre, (...) n'hésitèrent pas à se lancer au milieu d'une grêle de traits et à se jeter entre les combattants pour mettre fin au combat, pour mettre fin à la colère " (Tite-Live, I, 13, 1)6. Cette intervention aboutit à la paix et à la conclusion du traité fondateur du synœcisme : "on se tait, on s'apaise soudain. Puis, afin de conclure un traité, les chefs s'avancent. Non contents de faire la paix, ils réunissent en un seul les deux Etats...» (I, 13, 4). L'issue du conflit le fait apparaître rétrospectivement, et paradoxalement, comme une guerre civile d'avant la cité, à laquelle des femmes, seules, pouvaient mettre un terme, en tant que créatrices, dépositaires et symboles de la communauté pré-civique : la famille. Le plus important, de ce point de vue, est sans doute, plus encore que leur courage physique sur le champ de bataille, leur appel aux deux camps en tant que filles, épouses et mères ; «c'est pour nous que sont tués ou blessés nos maris et nos pères ; plutôt mourir que de survivre aux uns ou aux autres et de rester veuves ou orphelines » (I, 13, 3). Cette transmutation de l'entente familiale en concorde politique par la grâce des femmes trouve son emblème dans la décision de Romulus, chef politique et militaire : « quand il divisa la population en trente curies, ce fut leurs noms [ceux des Sabines] qu'il donna aux curies » (I, 13, 6).

11 2) Même conversion du privé au public, même rôle involontaire dans le déclenchement d'un conflit civil, même capacité d'initiative sacrificielle et politiquement fondatrice, quoique dans un contexte bien différent, avec l'épisode non moins légendaire du viol de Lucrèce. Femme de l'aristocrate Collatin, sous le règne tyrannique du dernier roi étrusque, Tarquin le Superbe, Lucrèce fait la preuve, face au soupçon entretenu envers les femmes - un thème essentiel à Rome, sur lequel nous allons revenir -, d'un comportement triplement exemplaire pour toute «matrone» romaine. Son maintien est celui, modeste, d'une épouse filant la laine, "veillant avec ses servantes et assise au milieu de sa maison» (Tite-Live, I, 57, 9) ; il contraste avec celui des «belles-filles du roi ", étendues - à l'étrusque... triste symbole de la dynastie !7 - sur des lits de banquet. Le viol par Sextus Tarquin lui donne, de manière sinistre, l'occasion de manifester deux autres vertus essentielles : la chasteté et la fidélité, castitas et fides. Si la première se dit en quelque sorte passivement, par son refus de céder aux avances de Tarquin et sa vaine tentative de résistance ( la passion de Tarquin triompha, si l'on peut dire, d'une vertu inébranlable », obstinatam pudicitiam, I, 58, 5), l'expression de la seconde est de la responsabilité et de la décision propres de Lucrèce. Celle-ci est très consciente de 
l'exemplarité de son geste : «pas une femme ne se réclamera de Lucrèce pour survivre à son déshonneur » $(\mathrm{I}, 58,10)$. Comme jadis les Sabines, c'est elle qui entraîne au-delà d'eux-mêmes les hommes venus la réconforter. Par sa mort volontaire, elle transmue l'agression personnelle en offense politique et donne le signal de la révolution dont Brutus prend la tête : «Par ce sang si pur avant le crime du prince, je jure devant vous, ô dieux, de chasser Lucius Tarquin le Superbe, lui, sa criminelle épouse et toute leur descendance (...) et de ne plus tolérer de rois à Rome, ni eux, ni aucun autre » (I, 59, 1). Décidément, dans une tradition historique hantée par le thème des fondateurs successifs, sur fond de déchirements internes, Lucrèce mérite d'être nommée, après les Sabines, la seconde fondatrice de Rome.

3) Les "premières femmes de la cité ", matronae primores ciuitatis (Tite-Live, XXIX, 14, 12), sont encore concernées dans l'épisode qui met en scène Claudia Quinta. L'expression valait d'être soulignée, car elle officialise dans le cadre civique l'existence d'une catégorie féminine représentative ${ }^{8}$. Nous ne sommes pas, cette fois, en période de guerre civile, puisqu'en 204 av. J.-C. la deuxième guerre punique bascule en faveur de Rome, et de Scipion face à Hannibal. Il n'en reste pas moins que l'intervention des « matrones ", sur décision du Sénat, a pour objet de refermer définitivement la période du doute, de l'angoisse et des discordes internes. Il s'agit de mettre un terme à l'« ère du soupçon »à l'égard des femmes qui hantait la mentalité collective depuis ces jours de 216 qui avaient vu l'écrasement des forces romaines par l'armée carthaginoise à Cannes, en Apulie. Parmi bien d'autres signes de la colère des dieux, deux Vestales, pour avoir failli à l'obligation de chasteté, avaient alors été condamnées au châtiment suprême. Claudia Quinta n'est pas une Vestale - quoique la tradition ne soit pas unanime sur ce point -, mais une matrone « de réputation douteuse » (Tite-Live, XXIX, 14, 12). L'important est qu'on l'ait choisie pour aller, au nom des mères romaines, accueillir l'« idole » d'une déesse mère, la Grande Mère Cybèle venue de Phrygie renforcer le panthéon de Rome. Surtout, c'est une authentique ordalie que, d'après Ovide (Fastes, IV, 247-348), Claudia a choisi de subir. Consulté, l'oracle de Delphes a en effet décrété que "quand Cybèle arrivera, elle devra être reçue par des mains chastes ». Arrivée à Ostie, port de Rome, la déesse hésite... Claudia est à la tête de la délégation, qui comprend « tous les chevaliers et, mêlés à la plèbe, les graves sénateurs... S'avancent à leurs côtés les mères, les filles et les brus, et les vierges qui veillent sur le foyer sacré ». Elle s'écrie : « si je ne suis pas coupable, tu te porteras par ton geste garante de mon innocence et, chaste, tu suivras de chastes mains ». Cybèle ayant dit oui, les femmmes la transportent à Rome. Et Claudia triomphe: "elle marche la première, faisant éclater la joie sur son visage, ayant enfin retrouvé, grâce au témoignage de la déesse, sa réputation de chasteté ». Ainsi l'épreuve personnelle déclenchée par la libre initiative d'une femme a-t-elle libéré de la suspicion toutes les femmes, garantissant à Rome entière, sous le patronage d'une Mère divine, le retour à la solidarité et à la confiance civiques .

4) Moins de vingt ans plus tard, avec l'affaire des bacchanales de $186^{9}$, d'autres femmes menacent de détruire l'œuvre de Claudia et la communauté unitaire, à la fois familiale et civique, qu'elle a symboliquement raffermie. Influencées par des courants dionysiaques actifs en Etrurie et en Italie du Sud hellénisée, notamment en Campanie, ces femmes sont elles aussi des "mères", mais des mères dévoyées. Elles aussi ont un leader: Paculla Annia, prêtresse venue de Capoue, n'hésite pas à bouleverser de son propre chef toutes les règles du culte. Les cérémonies, devenues nocturnes, mêleront désormais femmes et hommes, jeunes et vieux, libres et esclaves, dans une promiscuité - nous dit-on - où le vin et le sexe se donnent libre cours. Ainsi se constituent à Rome et 
en Italie des sortes de sectes d'un type radicalement nouveau, et radicalement inacceptable pour l'autorité romaine. Elles échappent au contrôle civique, qui est de règle à Rome pour toute manifestation de la vie religieuse. Elles s'organisent sous la direction de femmes, et non d'hommes. Elles comptent parmi leurs adeptes de nombreux fils de famille privés de père et, dirions-nous, de «surmoi » par les ravages de la deuxième guerre punique. Ces matres menacent en son cœur même le régime des patres lorsqu'elles font prêter à leurs « fils » un serment rituel perçu comme concurrent du serment militaire et civique habituellement prononcé devant Jupiter, au Capitole, par les jeunes gens en âge de devenir citoyens-soldats (17 ans). Aux yeux de la tradition romaine, ici encore exemplairement transmise par Tite-Live (XXXIX, 8-19), les initiatives de Paculla Annia, en ébranlant le « monisme » civico-familial (C. Gallini), ont failli remettre en cause ce qu'avaient progressivement construit (ou préservé) d'autres initiatives audacieuses, celles des Sabines, de Lucrèce, de Claudia Quinta.. Face à l'inquiétante démission des mères romaines, il est symbolique que la situation ait été sauvée par une affranchie d'origine étrangère, Hispala, courtisane amoureuse et généreuse qui, dénonçant la "conjuration ", s'est placée sous la protection d'une irréprochable mère de consul. Et l'on peut s'expliquer que le Sénat, engagé dans une guerre civile qui ne dit pas son nom, ait eu la main lourde: poursuites dans toute l'Italie, nombreuses arrestations, condamnations et exécutions de masse, interdiction de facto de célébrer le culte proscrit. Le moins symbolique, enfin, par le retour qui s'y marque à des usages archaïques, ne fut pas la remise des femmes coupables au pater familias pour le prononcé et l'exécution de la sentence.

Disposant désormais d'une suffisante "profondeur de champ ", nous pouvons revenir à la fin de la République et tenter de mettre en perspective par rapport à la tradition les portraits de femmes glanés chez Salluste puis, pour la phase ultérieure correspondant au déchaînement sans merci de la guerre civile, chez Cicéron et son biographe Plutarque, avant d'en recueillir une dernière image dans le marbre où fut, vers le même temps, gravé par un Romain pour nous anonyme l'éloge de son épouse, la «matrone inconnue ».

15 L'«audace virile » d'une Sempronia, le dérèglement systématique des mœurs et le renversement complet des valeurs qui lui sont attribués inscrivent cette femme dans la lignée des bacchantes, au point que plus d'une parenté est perceptible entre le récit de Salluste et la relation des Bacchanales par Tite-Live. Cette analogie de climat, cette commune démonisation des responsabilités féminines dans les maux de Rome ne doivent pas faire oublier des différences qui révèlent les évolutions survenues en cent vingt ans, et mettent au jour les spécificités de la situation de pré-guerre civile de 63. Dans le cas de Sempronia, on remarquera qu'au contraire de Paculla Annia, elle est pleinement romaine, aussi fortunée que cultivée; elle agit individuellement, elle n'a rien d'une prêtresse, son objectif avoué est de nature économique et politique.

16 Il serait encore moins pertinent de vouloir déceler de simples incarnations d'un rôle déjà écrit chez une Orestilla ou une Fulvia. Si la première est vantée par Catilina comme une épouse fidèle, elle offre naturellement peu d'autres points de comparaison avec Lucrèce - et pas uniquement parce qu'il lui manque et le pathétique d'une victime, et l'ardeur d'une combattante. Une de ses caractéristiques majeures - comme de ses compagnes «catiliniennes"-, c'est la richesse et la libre disposition de biens importants. Un même gouffre sépare les deux dénonciatrices : Hispala, au temps des bacchanales, est une étrangère, ancienne esclave et prostituée au grand cœur qui 
dénonce la «secte » par amour véritable et par sens du devoir. La figure de Fulvia, noble dévergondée incapable de tenir sa langue, ne présente aucun de ces traits spécifiques.

17 On serait donc tenté de dire des héroïnes malfaisantes, secondaires ou marginales de la période des guerres civiles que, si elles peuvent rappeler d'illustres précédents, elles se singularisent par deux tendances convergentes, bien caractéristiques de toute une époque : leur indépendance financière et familiale ${ }^{10}$ inouïe, leur indifférence absolue à l'égard de tout attachement, de tout idéal collectif - c'est-à-dire, à Rome, civique et gentilice. Tel est le grief fondamental que Cicéron fait articuler, dans sa plaidoirie de 56 av. J.-C. Pour Caelius, contre celle qui est pour lui et pour la postérité le modèle d'insulte à la tradition, la belle intrigante Clodia. Ce grief est mis dans la bouche de son ancêtre, l'illustre censeur Appius Claudius Caecus : «Femme, qu'as-tu à faire avec Caelius, un tout jeune homme (...) ? Issue du milieu le plus noble, unie à la plus illustre famille, pourquoi te lier si intimement avec Caelius? (...) Si les images des hommes de notre lignée ne te touchaient point, est-ce que mes descendantes au moins ne t'incitaient pas à rivaliser de vertus domestiques avec les gloires féminines de notre maison (aemulam domesticae laudis in gloria muliebri), la noble Claudia Quinta ou la vestale Claudia, qui, serrant son père dans ses bras, ne laissa pas (passa non est) son adversaire, le tribun de la plèbe, l'arracher à son char, lors de son triomphe...? " (XIV, 34). Nous avons déjà reconnu les vertus de la première nommée. Il reste vrai, certes, que la référence étroitement familiale, "gentilice ", la valorisation d'une vierge vestale et l'assimilation de la gloria muliebris à la domestica laus paraissent réduire l'idéal proposé à un état de soumission quotidienne. Mais il est symptomatique que Clodia soit interpellée au nom d'héroïnes du temps jadis, de celles qui avaient fait admirer au premier chef leur esprit de décision et leur refus de subir: le verbe passae non sunt..., "elles n'ont pas toléré", peut valoir pour les Sabines et pour Lucrèce comme pour Claudia Quinta .

Gardons-nous donc de mutiler ou de déformer à l'excès l'image du surmoi féminin opposé et proposé aux "déviantes» de la République finissante. Il ne leur est pas enjoint de se borner à filer la laine. Ce qui leur est finalement reproché n'est pas tant de prendre des initiatives que de les prendre hors de tout cadre civique et familial, hors de toute référence à la tradition, à partir d'une condition sexuelle et économique parfaitement indépendante. En un mot, leur crime est moins d'être des femmes bien romaines que, comme trop de leurs contemporains mâles, des femmes de leur temps: le temps des guerres civiles.

Il n'est pourtant pas impossible de rencontrer, au cœur de tels événements, quelques " héroïnes positives " présentant une combinaison inédite des vertus d'autrefois et des capacités nouvelles acquises par les femmes. De ce dernier cas, nous retiendrons en premier lieu l'exemple de Térentia, déjà nommée. L'épouse de Cicéron a fortement poussé son mari, jadis (sans doute pour des raisons de jalousie personnelle), à s'attaquer à Clodia. Plus tard, au temps de la guerre entre César et Pompée, Térentia apparaît à la fois, à travers la correspondance de Cicéron, comme la confidente, la gestionnaire attentive des biens ${ }^{11}$, mais aussi comme une informatrice et parfois une conseillère politique - à défaut d'une partenaire intellectuelle,$-{ }^{12}$ comme une charge enfin, et une préoccupation dont il arrive que Cicéron fasse part à Atticus et à d'autres amis : "que faire de Térentia et de notre fille? Les laisser à Rome ou les mettre à l'abri ?» Cette question récurrente des années 50-49 - moment de l'affrontement final entre César et Pompée - est bien révélatrice, sur un plan général, de la situation d'une 
épouse d'homme public en ces temps de guerre civile: si elle est restée à la Ville pendant que son mari est ballotté ici et là par les aléas du conflit, elle est précieuse comme garante de continuité du patrimoine et source de renseignements sur l'évolution du " centre du pouvoir »; lorsque les choses se gâtent, sa sécurité et celle de la famille passent au premier plan. Cicéron, toutefois, a des raisons plus personnelles d'attendre beaucoup de Térentia en de telles circonstances.

Nous connaissons par Plutarque (Vie de Cicéron, 17) le rôle qu'avait joué cette femme, aux côtés de quelques nobles dames et surtout des Vestales, à cet instant précis et dramatique de l'année 63 où le consul Cicéron hésitait à faire condamner à mort les complices de Catilina, démasqués et emprisonnés. L'apparition soudaine d'une flamme brillante parmi le feu vacillant de l'autel fut par elles interprété comme un "prodige ", et utilisé par Térentia pour décider son mari à une sévérité sans réserve. Quelles leçons tirer de cet épisode (qu'il ait ou non réellement eu lieu, peu importe : il a été rapporté comme plausible)? La première est que les femmes des principaux magistrats n'accompagnent pas seulement, à titre obligatoire et en somme décoratif, des cérémonies traditionnelles sans prise sur les événements. La seconde, que les Vestales, de leur côté, ne sont pas cantonnées à un rôle symbolique et quelque peu désuet. Tout au contraire : les unes ont choisi collective-ment une option dans le conflit en cours, et pèsent par tous les moyens sur les décisions des hommes. Quant aux secondes, elles disposent d'une marge de manœuvre et d'une liberté d'interprétation, si ce n'est de mise en scène, au cœur même de ce qui fait leur vocation : l'entretien du feu sacré de Rome. Les unes et les autres, on le voit, ont de qui tenir. Un Appius Claudius d'esprit "moderne » ne les aurait pas désavouées, et les conspiratrices de type catilinien trouvent en face d'elles, symboliquement et réellement, des adversaires du même sexe qui ne sont pas moins qu'elles « à la hauteur » des guerres civiles.

21 À côté de ces grandes dames, une place revient, aussi discrète qu'éminente, à une «matrone inconnue », leur contemporaine, longtemps nommée Turia par les érudits modernes. Son éloge funèbre, la laudatio composée par son mari, nous a été transmis par une longue inscription sur marbre en provenance de Rome ${ }^{13}$. Bien entendu, on y porte au pinacle « ses qualités domestiques, sa vertu, sa docilité, sa gentillesse, son bon caractère, son assiduité aux travaux de la laine... ", bref, comme le souligne lui-même l'auteur de l'éloge, «toutes les autres vertus innombrables qu'ont toutes les matrones soucieuses d'une bonne renommée » (Eloge, I, 30-33). Mais l'essentiel n'est pas dans ces banalités: débordant les qualités habituellement attendues de son sexe, l'épouse glorifiée s'est montrée habile procédurière pour défendre victorieusement un héritage contesté (I, 18-25) ; quand son mari était en fuite après la défaite à Pharsale, en 49 , des partisans de Pompée, elle lui a fourni par la ruse, et mue par un dévouement sans limite, ses bijoux, des esclaves, de l'argent, de quoi se subvenir (II, 2 a); face aux hommes de main d'un chef de bande (Milon), elle a protégé avec succès la demeure familiale (II, 9 a) ; c'est à ses « informations rapides » et à ses « conseils » que son mari, comme il le confesse, a «dû d'éviter des malheurs présents et imminents et de conserver la vie » (II, 5). Elle est allée jusqu'à braver le triumvir Lépide - un leader bien typique de guerre civile - en venant, au prix d'humiliantes vexations, lui réclamer de rétablir son époux dans ses droits (II, 11-21). En un mot, la riche héritière qui défend ses biens autant qu'elle les gère, avant de les sacrifier aux impératifs de la lutte, la femme de tête et de poigne qui sauve sa domus, puis un mari qu'elle ne cesse de renseigner et de conseiller, cette matrone exemplaire a su faire face, autant et mieux qu'homme romain, aux situations les plus graves que peut engendrer une guerre civile. 
Ses actes peignent les vertus que requérait l'instant, et que l'Éloge laisse innommées : courage, habileté, ténacité, intelligence des situations, capacité d'initiative et d'influence sur le cours des choses. L'admiration reconnaissante de son époux mérite ainsi le mot de la fin: «ce que je veux mettre en valeur, ce sont les vertus qui ne sont qu'à toi, dont peu d'hommes ont rencontré les pareilles, des vertus qui donnent la force de supporter de telles épreuves et de rendre de tels services, car le destin des hommes a pris soin que de telles conjonctures fussent rares » (Eloge, I, 34-36).

\section{BIBLIOGRAPHIE}

BOISSIER, G.

1910 Cicéron et ses amis, Paris.

DURRY, M.

1950 Eloge funèbre d'une matrone romaine, Ed., trad. et comm. M. Durry, Paris, CUF.

JAL, P.

1963 La guerre civile à Rome. Etude littéraire et morale de Cicéron à Tacite, Paris.

MOREAU, Ph.

1982 Religio clodiana. Un procès politique en 61 av. J.-C., Paris.

PAILLER, J.-M.

1988 Bacchanalia, Rome.

PAILLER, J.-M.

1995 « Marginales et exemplaires. Remarques sur quelques aspects du rôle religieux des femmes dans la Rome républicaine ", Clio, Histoire, Femmes et Sociétés, 2, p. 41-60.

\section{NOTES}

1. Perception, rôle : insistons bien. Ni le texte de Salluste, ni d'autres documents ne nous permettent de dépasser le stade d'une analyse au second degré, d'un commentaire de l'image. Cette image est finalement moins de l'ordre de la représentation personnelle que du rôle prêté à une « classe » déterminée d'individus dans un type de situation donné.

2. De ce renversement témoigne encore chez Salluste le mot prêté à Caton : «il y a longtemps que nous avons perdu le véritable sens des mots... Voilà pourquoi la République touche à sa perte » (Catilina, LII, 11). Cf. Jal, $1963:$ 460-473 (ce livre « classique » et toujours utile sur la guerre civile à Rome porte bien sa date : sur plus de cinq cents pages, à peine trois ou quatre sont consacrées au rôle des femmes...). 3. Les traductions de Salluste citées ici sont dues à A. Ernout et empruntées à la Collection des Universités de France (éditions Budé). 
4. Cf. Moreau, 1982.

5. Nous soulignons cette mention, bien caractéristique de la puissance et de l'autonomie financières acquises à cette époque par certaines femmes.

6. Trad. G. Baillet (CUF).

7. Une dynastie qui, avec la « faiseuse de rois » Tanaquil, femme de Tarquin l'Ancien, et Tullia, l'abominable épouse de Tarquin le Superbe (un personnage de muliebri audacia selon Tite-Live, I, 46, 6...), a fourni deux « anti-modèles » idéaux aux intrigantes de la fin de la République. Cf. Jal, 1963 : 353. Bien entendu, l'analyse du processus est réversible : contemporain des guerres civiles, Tite-Live prête aux reines étrusques $l^{\prime} \ll$ audace » de Sempronia et de ses pareilles.

8. Cf. Pailler, 1995 : 41-60.

9. Cf. sur l'ensemble de l'épisode Pailler, 1988.

10. En même temps - mais cela n'a rien de contradictoire dans la Rome de cette époque - leurs attaches les relient à un même univers très restreint, où les circonstances leur font jouer tour à tour le rôle d'espionnes, d'otages ou d'intermédiaires. Cf. Jal, 1963 : 328-329.

11. Ces biens n'étaient pas modestes : sa dot, selon Plutarque (Vie de Cicéron, 8), s'était élevée à « 120000 drachmes »; elle possédait des maisons à Rome, une forêt près de Tusculum (Cicéron, A Atticus, II, 4). Son âpreté au gain fut aux yeux de certains une cause majeure du divorce des époux (Boissier, 1910 : 99-101). Une chose est sûre : en cette circonstance, Cicéron se refusa à une querelle d'intérêt avec elle, lui faisant accorder tout ce qu'elle demandait (A Atticus, XII, 22). Délicatesse ? Peut-être. Lassitude, au milieu des priorités de l'heure ? Sans doute. Mais plus sûrement infériorité reconnue en matière de gestion domestique, hommage implicite rendu à la femme d'affaires par l'homme d'Etat et l'intellectuel.

12. En matière religieuse, par exemple, il lui indique, désinvolte, qu'il se consacre au bonheur des hommes avec autant de soin qu'elle met de vénération à honorer les dieux (Ad Fam., XIV , 4). De manière plus positive, l'auteur de l'Eloge analysé ci-dessous félicitera son épouse de sa " piété sans superstition » (I, 31).

13. Eloge funèbre d'une matrone romaine, Ed., trad. et comm. Durry, 1950 (nous apportons quelques modifications à cette traduction). Résumant sa carrière « héroïque ", M. Durry qualifie joliment et justement la destinataire de cette louange d'« amazone des guerres civiles» (Introduction, p. XCIV) ; cf. Jal, 1963 : 353.

\section{RÉSUMÉS}

Les femmes occupent dans les récits des guerres civiles de Rome une place importante, parfois présentée sous un jour favorable. Le fait peut surprendre, dans une société aussi patriarcale et dans des récits tournés vers l'exaltation d'une tradition on ne peut plus virile. On y dénonce les aventurières, telle Sempronia, qui entourent, dans les troubles du I ${ }^{\text {er }}$ s. av. J.-C., les adversaires de la République, et au premier rang un Catilina. Mais l'« audace toute virile » qui leur est prêtée, si elle représente dans leur cas une perversion, une inversion des valeurs condamnée sans réserve, peut ailleurs se réclamer d'exemples plus nobles. Trois exemples positifs et plus ou moins 
légendaires (les Sabines, Lucrèce, Claudia Quinta), un contre-exemple historique (les Bacchantes) illustrent la manière dont les «rôles " féminins de la fin de la République s'adossent à une tradition aussi riche que nuancée. Finalement, si la femme sait se mettre au service de la famille et de la cité, sa capacité d'initiative représente une chance de salut pour tous. Tel est l'exemple proposé par la «matrone inconnue » dans l'éloge funèbre qu'a fait graver dans le marbre, au temps de Cicéron, un époux admiratif et reconnaissant.

In the ancient accounts of the Civil Wars at Rome, an important space is devoted to the rôle played by women a rôle which, in some cases, is actually described as helpful. This may seem surprising, as we are dealing with a patriarcal society and with accounts which generally reflect and exalt a virile tradition. The authors denounce intriguing women, such as Sulpicia, who surround those who are threatening the Roman Republic, and among them, in the first place, Catilina. But their so-called uirilis audacia may well, in their case, represent a perversion, an inversion of social values which is entirely to be blamed; it remains that in some other, different cases, such an audacious behaviour is actually based on more illustrious examples. Among them are examined those, more or less legendary, of the Sabine women, of Lucretia, of Claudia Quinta, and the counterexample of the historic Bacchantes. The four of them make clear the way in which the feminine roles, at the end of the Republic, rely upon a rich and diversified tradition. Finally, if Roman women decide to serve their city and family, their ability to take the initiative may prove very useful to every people around them. Such is the example of an unknown lady, whose memory is highly praised, by the time of Cicero, in a marble inscription composed with admiration by her grateful husband.

\section{AUTEUR}

\section{JEAN-MARIE PAILLER}

Jean-Marie PAILLER, Professeur d'Histoire Ancienne à l'Université de Toulouse II le Mirail. Domaines d'étude : La religion antique, à Rome et en Gaule, mais aussi en Grèce et en Etrurie, notamment ce qui touche à Dionysos-Bacchus. Etudes en cours sur Vesta et les Vestales. Histoire de la culture et de l'éducation dans l'Antiquité. Histoire des femmes à Rome et en Gaule romaine. Histoire et archéologie de la Gaule romaine, notamment dans le sud-ouest de la Gaule.

Publications : Nombreux articles sur les sujets précédents ; une contribution au numéro de Clio sur "Femmes et religions". Parmi les ouvrages : Actualité de l'Antiquité, Paris, CNRS, 1989 (J.-M. Pailler éd.) ; Bacchanalia, Rome, 1988 (Ecole Française de Rome) ; Bacchus. Figures et pouvoirs, Paris, Belles-Lettres, 1995. 